\title{
Challenges in the design of multitarget drugs against multifactorial pathologies: a new life for medicinal chemistry?
}

"While there is a research of modern phenotypic assays, network pharmacology deals with the second problem, namely the proper selection of the targets."

Keywords: anticancer compounds = medicinal chemistry $\approx$ multitarget drugs $\approx$ network pharmacology

The medicinal chemistry field is in a period of crisis, mirrored in the low number of new drug approvals, especially considering the parallel increasing levels of investments in research. This happens when, at the same time, combinatorial chemistry and high-throughput screening increased the number of tested substances and the redutionistic approach (the magic bullet against a target assayed in in vitro test systems) was followed for the development of new drugs, in order to reduce promiscuity (off-target interactions), which was linked to the appearance of side effects [1].

However, this approach proved to be only partially successful, since, for first-in-class molecules approved by the US FDA between 1999 and 2008, a period of time in which the target-directed strategy was the main approach for new pharmacologically active compounds, $37 \%$ resulted from projects that used phenotypic screening and only $23 \%$ from target screening. This statistic is all the more startling considering the wider popularity of target screening with respect to phenotype [2]. At the same time, it is becoming clear that many drugs may derive their therapeutic action (and/or their off-target activities) from the interactions with multiple proteins; for noncancerous diseases, the average number of interaction partners for protein targets of marketed drugs is 4.24 , and for cancer targets it is 7.82 [3]. Thus, it looks that many clinically useful drugs are multitarget agents.

In fact, except for diseases caused by mutation or elimination of specific genes, many others for example, cancer, metabolic disorders and CNS diseases, are multifactorial; it is becoming clear that disease states are resistant to perturbations and maintain their state through mechanisms such as back-up circuits and fail-safe mechanisms. Moreover, cancer and infectious diseases are characterized by the appearance of resistance, owing to the selection of one or more mutations in encoded target proteins [4-6]. These findings give the rationale for the use of drugs able to target multiple, unrelated proteins. Accordingly, drug associations gained popularity as therapeutic, multitarget agents [7].

Medicinal chemistry research addressed this point by the design of multitarget agents. The procedure of the discovery of these agents was based on in vivo assays or the design of assembled pharmacophores of the target ligands under consideration, with or without the aid of computational techniques. These procedures were demonstrated to be successful in some disease areas; some of the compounds thus obtained entered clinical trials (e.g., drugs used to treat psychiatric disorders), but few compounds became clinically available [7]. Perhaps the most successful example of recently discovered multi-target drugs is kinase inhibitors as anti-angiogenesis anticancer agents. Achieving a durable and efficient antiangiogenic response requires approaches to simultaneously or sequentially target multiple aspects of the tumor microenvironment [8]. In this case, it is relatively easy to obtain a drug-like inhibitor, since kinases (more than 500) share a very similar binding site for ATP, which becomes the binding site of the inhibitors; the problem in this case is to obtain selectively nonselective compounds using the subtle differences in the binding sites.

The development of sunitinib, one of the first multikinase inhibitors approved (2006), started as a selective VEGFR2 inhibitor drug, but during the project evolution, the target was broadened since it was hypothesized that simultaneous inhibition of multiple RTK, critical to regulation of both tumor proliferation and angiogenesis, would cooperate to produce anti-tumor efficacy.

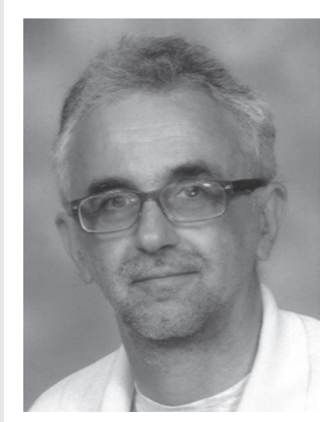

Luca Costantino

Author for correspondence: University of Modena e Reggio Emilia, Dipartimento di Scienze Farmaceutiche, Via Campi 183, 41100 Modena, Italy

Tel.: +390592055125

Fax: +39059 2055131

E-mail: luca.costantino@unimore.it

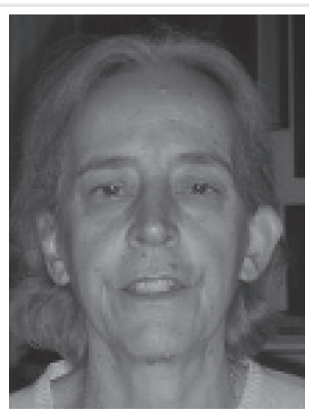

Daniela Barlocco

University of Milano, Dipartimento di Scienze Farmaceutiche,

Via L. Mangiagalli 25,

20133 Milano, Italy 
The product was optimized through action on VEGFR and PDGFR kinases [9], but after it was shown that its selectivity depends on the number of kinases tested, a characteristic shared by almost all known designed (multi) kinase inhibitors. When tested on all kinases available (more than 400 , KINOMEscan ${ }^{\circledR}$ platform), sunitinib showed a score $S$ of 0.569 at $3 \mu \mathrm{M}$ concentration and of 0.183 at $100 \mathrm{nM}$ (S: ratio between the number of kinases found to bind to the drug with $\mathrm{K}_{\mathrm{d}}$ less than $3 \mu \mathrm{M}$ or $\mathrm{K}_{\mathrm{d}}$ less than $100 \mathrm{nM}$, and the total number of kinases tested); thus, a great number of kinases are inhibited by this drug [10], and the compound appears to actually be a nonselective multitarget compound. This compound demonstrated that it was able to make some tumors more aggressive in animal models $[11,12]$; this is probably due to the drug-induced hypoxia that activates the Akt/ $\beta$-catenin pathway which, in turn, stimulates the growth of specific, more aggressive, cancer cells populations. A solution that has been hypothesized to overcome this problem is represented by the combination (coadministration) of anti-angiogenic inhibitors with drugs able to block the cancer cell response to hypoxia or with compounds able to block Akt/ $\beta$-catenin pathway [13]. Thus, only a full understanding of the networks present in a cancer cell can aid in the selection of the appropriate targets to fight this, and other, complex, multifactorial diseases. Another study suggested that the off-target effects of the RTK inhibitor could be responsible for the disruption of the homeostatic barrier function of the host vasculature, with the consequent metastasis promotion observed [14].

\section{"Medicinal chemistry research addressed this point by the design of multitarget agents. The procedure of the discovery of these agents was based on in vivo assays or the design of assembled pharmacophores of the target ligands under consideration, with or without the aid of computational techniques."}

Drug design using modern procedures, such as the fragment-based approach, do not guarantee that off-target activities are absent: sorafenib, a multikinase inhibitor discovered following this strategy [15], was recently shown to be a nanomolar antagonist of the 5-HT receptors [16]. This issue probably will be successfully reduced in the future by means of in silico procedures, since computational chemistry is developing strategies to predict the promiscuous binding propensities of drug molecules [16].

Thus, the available technologies are able to allow the design of clinically useful multitarget agents $[7,17]$, but these procedures have to be integrated with phenotype screening [18] and a proper selection of targets relevant for a given pathology. Problems linked on relying only on in vitro assays have been considered in the development of multikinase inhibitors: translating the isolated enzyme inhibition to efficacy against the native kinase needs cell-based assays in order to take into account intracellular ATP concentrations, membrane permeability, presence of phosphatase enzymes and the relative concentrations of the kinase targets and their substrates [10].

\section{"This issue probably will be successfully reduced in the future by means of in silico procedures, since computational chemistry is developing strategies to predict the promiscuous binding propensities of drug molecules."}

While there is a research of modern phenotypic assays [18], network pharmacology deals with the second problem, namely the proper selection of the targets. Cellular activity and response following the interaction of molecules with biological targets comprises interaction with multiple components (networks: proteinprotein interactions, protein-DNA interactions, epigenetic changes, together with changes in metabolic and functional pathways); these interactions are networked at the organ and subsequently at the organism level. Network pharmacology targets the myriad of interaction networks between molecules into cells, instead of targeting a single protein; systems biology, in turn, studies how the sum of all interactions between the molecules in a cell creates a greater whole [19]. This field has already revealed a number of promising examples of rational and network-based drug-target predictions, successfully tested also in vivo [7,19].

At least two companies are grounded on network pharmacology to find out new drugs. E-therapeutics, with its proprietary network pharmacology platform, is able to analyze network of proteins associated with a particular disease; in this way, it is possible to identify a disease signature, proteins in the network at which intervention would have maximum impact. The scientists mapped out 103 protein networks that help cancer cells to evade 
apoptosis, then selected a drug, previously known as safe but ineffective in Phase III studies, dexanabinol, able to modulate the network (a sort of drug rescue, in which promising compounds that have been developed for one indication but have failed to reach the clinic are redirected toward another indication). The product showed to be active on cancer cell lines and it is now in Phase I clinical studies for the treatment of glioma. Another company, Pharnext, is studying in Phase II PXT3003, a combination of the GABA agonist, baclofen, opioid receptor antagonist, naltrexone and sorbitol, as a therapeutic treatment for neuropathic

\section{References}

1 Scannell JW, Blankley A, Boldon $\mathrm{H}$ et al. Diagnosing the decline in pharmaceutical R\&D efficiency. Nat. Rev. Drug Discov. 11(3), 9 191-200 (2012).

2 Swinney DC, Anthony J. How were new medicines discovered? Nat. Rev. Drug Discov. 10(7), 507-519 (2011).

3 Hase T, Tanaka H, Suzuki Y et al. Structure of protein interaction networks and their implications on drug design. PLoS Comput. Biol. 5, e1000550 (2009).

4 Kamb A, Wee S, Lengauer C. Why is cancer drug discovery so difficult? Nat. Rev. Drug Discov. 6(2), 115-120 (2007).

5 Marusyk A, Almendro V, Polyak K. Intratumor heterogeneity: a looking glass for cancer? Nat. Rev. Cancer 12(5), 323-334 (2012).

6 Barbachyn MR. Recent advances in the discovery of hybrid antibacterial agents. Ann. Rep. Med. Chem. 43, 281-290 (2008).

7 Costantino L, Barlocco D. Designed multiple ligands: basic research vs clinical outcomes. Curr. Med. Chem. 19(20), 3353-3387 (2012).

Charcot-Marie-Tooth disease [20]. If clinically successful, network pharmacology surely will heavily modify the drug-discovery process.

\section{Financial \& competing interests disclosure}

The authors have no relevant affiliations or financial involvement with any organization or entity with a financial interest in or financial conflict with the subject matter or materials discussed in the manuscript. This includes employment, consultancies, honoraria, stock ownership or options, expert testimony, grants or patents received or pending, or royalties.

No writing assistance was utilized in the production of this manuscript.

8 Weis SM, Cheresh DA. Tumor angiogenesis: molecular pathways and therapeutic targets. Nat. Med. 17(11), 1359-1370 (2011).

9 Sun CL, Christensen JG, McMahon G. Discovery and development of sunitinib (SUI11248): a multitarget tyrosine kinase inhibitor of tumor growth, survival, and angiogenesis. In: Kinase Inhibitor Drugs. Li R, Stafford JA (Eds). Wiley, NJ, USA, 3-40 (2009).

10 Swarbrick ME. The learning and evolution of medicinal chemistry against kinase targets. In: Kinase Drug Discovery. Ward RA, Goldberg F (Eds). Royal Society of Chemistry, Cambridge, UK, 79-95 (2012).

11 Paez-Ribes M, Allen E, Hudock J et al. Antiangiogenic therapy elicits malignant progression of tumors to increased local invasion and distant metastasis. Cancer Cell 15(3), 220-231 (2009).

12 Ebos JML, Lee CR, Cruz-Monoz W et al. Accelerated metastasis after short-term treatment with a potent inhibitor of tumor angiogenesis. Cancer Cell 15(3), 232-239 (2009).

13 Casanovas O. Limitations of therapies exposed. Nature 484(4) 44-46 (2012).
14 Chung AS, Kowanetz M, Wu X et al. Differential drug class-specific metastatic effects following treatment with a panel of angiogenesis inhibitors. J. Pathol. 227(4), 404-416 (2012).

15 Erlanson DA. Fragment-based drug discovery of kinase inhibitors. In: Kinase Inhibitor Drugs. Li R, Stafford JA (Eds). Wiley, NJ, USA, 461-484 (2009).

16 Lin X, Huang XP, Chen G et al. Life beyond kinases: structure-based discovery of sorafenib as nanomolar antagonist of 5-HT receptors. J. Med. Chem. 55(12), 5749-5759 (2012).

17 Bottegoni G, Favia AD, Recanatini M et al. The role of fragment-based and computational methods in polypharmacology. Drug Discov. Today 17(1-2), 23-34 (2011).

18 Lee JA, Uhlik MT, Moxham CM et al. Modern phenotypic drug discovery is a viable, neoclassic pharma strategy. J. Med. Chem. 55(10), 4527-4538 (2012).

19 Klipp E, Wade RC, Kummer U. Biochemical network-based drug-target prediction. Curr. Opin. Biotechnol. 21(4), 511-516 (2010).

20 Network pharmacology moves into the clinic. Nat. Rev. Drug Disc. 11(8), 589 (2012). 